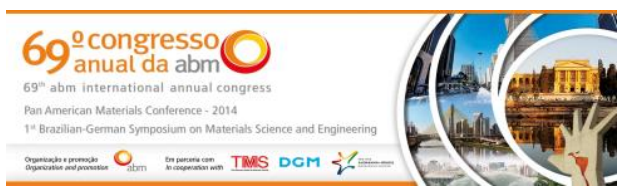

Tema: Engenharia de Superfície

\title{
ESTUDO DA INFLUÊNCIA DOS PARÂMETROS SOBRE A ESPESSURA DE REVESTIMENTO DEPOSITADO POR ASPERSÃO TÉRMICA POR CHAMA A PÓ*
}

\author{
Paulo Sergio Olivio Filho ${ }^{1}$ \\ Émillyn Ferreira Trevisani Olivio² \\ Gustavo Bavaresco Sucharski ${ }^{3}$
}

\section{Resumo}

A aspersão térmica é utilizada para produzir revestimento quando há necessidade de aumentar a vida útil de sistemas e peças, podendo ser também utilizada na recuperação delas, em alguns casos. Com o intuito de compreender melhor o processo de aspersão térmica por chama a pó, o objetivo deste trabalho é analisar os fatores influentes no aumento de espessura depositada pelo processo, utilizando o delineamento de experimento de Taguchi para avaliar os fatores e níveis dos fatores que geram as melhores condições de aplicação para o revestimento. Para isto, foram escolhidos três fatores de controle com três níveis cada um, dos quais foi possível seu controle em laboratório. A deposição foi realizada em chapas de aço SAE1020, e revestida com pó de alumínio não atomizado. A seção transversal dos revestimentos foi analisada por microscópio ótico. Os resultados dos experimentos foram analisados através de estatística descritiva, gráfica e análise pelo delineamento de Taguchi, baseada na análise de Sinal/Ruído. Sendo evidente que os fatores do processo são estatisticamente significativos: número de passes, fluxo de nitrogênio e a taxa de alimentação de pó. Esta análise se mostrou eficaz, gerando os resultados esperados para otimização dos parâmetros do processo.

Palavras-chave: Aspersão Térmica; Revestimento; Taguchi; Alumínio.

\section{STUDY ON THE INFLUENCE OF PARAMETERS ON THE THICKNESS OF COATING DEPOSITED BY POWDER FLAME SPRAY}

\section{Abstract}

Thermal Spray is used to produce coatings, when there is a need in increasing the lifetime of systems and parts, and in some cases, it can be used in recovering them. In order to better comprehend the Flame Spray process, this paper aimed to analyze the influent factors in the increase of the thickness, using the Taguchi design of experiment to evaluate the factors and levels of factors that create the best conditions for applying the coating thermally sprayed. For this cause were chosen three control factors with three level each one, which were controlled in laboratory. The deposition was performed in SAE1020 steel plates, with nonatomized aluminum powder. The cross section of the coatings were analyzed by optical microscope. The experimental results were analyzed using descriptive statistics, graphical analysis and design by Taguchi, based on analysis of signal/noise ratio. As evident that the process factors are statistically significant: the number of passes, and the nitrogen flow rate of powder feed. This analysis was effective, generating expected results for optimization of process parameters results.

Keywords: Thermal Spray; coating; Taguchi; Aluminum.

1 Engenheiro de Produção, Bacharel, Analista, Engenharia Processos, Segula do Brasil, Curitiba, PR, Brasil.

2 Tecnóloga em Manutenção Mecânica Industrial, Mestra, Professora, COMIM, UTFPR, Cornélio Procópio, PR, Brasil.

3 Tecnólogo em Eletromecânica, Mestre, Doutorando, DEMEC, UFPR, Curitiba, PR, Brasil.

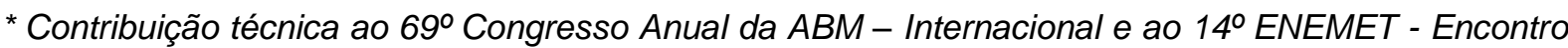
Nacional de Estudantes de Engenharia Metalúrgica, de Materiais e de Minas, 21 a 25 de julho de 2014, São Paulo, SP, Brasil.
} 


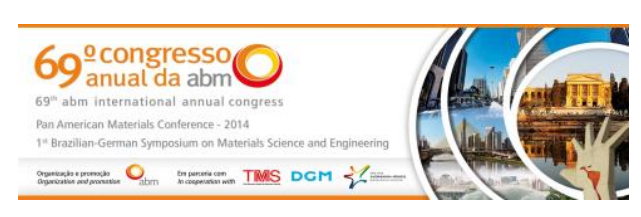

\section{INTRODUÇÃO}

Nas ultimas décadas os conceitos estatísticos e de delineamento de experimentos têm ganhado importância crescente no cotidiano das organizações [1]. Para se obter os melhores parâmetros e níveis de processo que otimizem os resultados desejados, o uso dos conceitos de planejamento de experimentos é indispensável, a fim de identificar as causas que provocam as falhas ou as perdas de qualidade no produto e no processo de fabricação, definindo ações de melhoria mais eficazes nos parâmetros e níveis do processo.

Os processos especializados de aspersão térmica têm larga utilização tanto na fabricação quanto na manutenção de peças e equipamentos. A natureza dos processos de aspersão térmica é sinérgica. Os números de componentes e variáveis envolvidas são grandes e quando escolhidos adequadamente em conjunto, e devidamente aplicados, podem produzir maior efeito do que quando considerados individualmente. No entanto, cada componente e variável devem ser bem compreendidos, a fim de permitir sua escolha e operação adequada em cada processo em particular [2].

Todavia, pouco se sabe sobre a influência das variáveis da máquina e processo na busca por maior espessura de revestimento depositado, não sendo encontrados estudos e dados do fabricante Sulzer Metco [3] para este fim. Para otimizar o processo de fabricação, e obter uma melhor compreensão dos parâmetros do processo, esse trabalho pode contribuir para a redução de tempo de processo e custos de fabricação, além de um melhor controle na espessura que se deseja obter para cada componente aspergido. Para isso se faz necessário uma análise estatística adequada das variáveis controláveis e que realmente influenciam na espessura do revestimento de aspersão térmica por chama pó.

\section{MATERIAIS E MÉTODOS}

\subsection{Definição dos Materiais e Equipamentos de Processo}

Os materiais e equipamentos utilizados no processo de aspersão térmica por chama a pó para análise e experimentação são:

a) Uma unidade de controle de aspersão térmica por chama a pó Sulzer Metco ${ }^{\circledR}$ e uma pistola de aspersão térmica por chama a pó Sulzer Metco® 6P-II-A;

b) Sistema de controle de posição e velocidade de passes;

c) Máquina de jateamento para limpeza dos substratos;

d) Metal de adição;

e) Substrato para revestimento.

\subsubsection{Equipamento de aspersão térmica a chama a pó}

As deposições foram realizadas no Laboratório de Aspersão Térmica e Soldagens Especiais (LABATS) do Departamento de Engenharia Mecânica (DEMEC) da UFPR. Foi utilizada a cabine de aspersão com sistema de exaustão e retirada do pó via parede com lâmina de água; equipamento de aspersão térmica Sulzer, controle de alimentação de pó modelo 5MPE com pistola 6PII e alimentador RP5, funil por onde passa o pó com alimentação constante. Os gases utilizados no processo de aspersão foram oxigênio e acetileno, e para gás de arraste nitrogênio, a Tabela 1 mostra os parâmetros pré-fixados pelo fabricante.

\footnotetext{
* Contribuição técnica ao $69^{\circ}$ Congresso Anual da ABM - Internacional e ao 14ํㅡㄹ ENEMET - Encontro Nacional de Estudantes de Engenharia Metalúrgica, de Materiais e de Minas, 21 a 25 de julho de 2014, São Paulo, SP, Brasil.
} 


\subsubsection{Materiais}

Como substrato, foram utilizadas chapas de aço carbono SAE 1020, cortadas nas dimensões 100X25X1,25mm.

Como metal de adição foi utilizado o pó de alumínio (Al) não atomizado com dimensão de \#140 mesh (105 microns) e pureza de 99,5\%, especificado pela Norma Petrobrás N 2568, 2011.

\subsection{Definição das Variáveis de Entrada}

Para se definir as variáveis de entrada ou parâmetros de processo do experimento, foi realizado um brainstorm a fim de determinar os fatores que poderiam influenciar no aumento da espessura de revestimento aspergido. Abordagens semelhantes em outro processo já foram realizadas por Syrcos [4], Wu e Chang [5].

As variáveis de entrada influenciante no processo a partir do brainstorm foram:

- Fluxo de gás de arraste (Fluxo de Nitrogênio);

- Pressão do gás arraste;

- Taxa de Alimentação de pó;

- Velocidade do passe;

- Número de passes;

- Relação de combustão (Oxigênio/Acetileno);

- Temperatura de pré-aquecimento do substrato;

- Distância Pistola/Substrato.

As variáveis escolhidas para análise foram: Número de passes, Taxa de alimentação de pó e Fluxo de gás de arraste (Fluxo de nitrogênio).

\subsection{Definição do Arranjo Ortogonal}

A partir da escolha dos 3 fatores, foram designados 3 níveis para cada um deles, gerando assim a matriz de experimentos vista na Tabela 4. Desta forma, foi utilizado uma matriz ortogonal L9, com nove rodadas de experimentos e três réplicas de cada uma, por se tratar de um arranjo experimental com o menor número de experimentos possíveis para os três fatores.

Tabela 4. Fatores e Níveis dos Experimentos

\begin{tabular}{c|l|ccc}
\hline \multicolumn{2}{c|}{ Fatores } & \multicolumn{3}{c}{ Níveis } \\
\multicolumn{2}{c|}{} & 1 & 2 & 3 \\
\hline A & Numero de Passes & 4 & 8 & 12 \\
\hline B & $\begin{array}{l}\text { Taxa de alimentação } \\
\text { (g/min) }\end{array}$ & 30 & 40 & 50 \\
\hline C & Fluxo de Nitrogênio (SCFH) & 10 & 12,5 & 15 \\
\hline
\end{tabular}

Na Tabela 5 é mostrada a matriz de combinação de níveis de parâmetros nos nove experimentos, juntamente com a sequência experimental gerada aleatoriamente, através de software para a realização dos experimentos.

\footnotetext{
* Contribuição técnica ao 69ํ Congresso Anual da ABM - Internacional e ao 14ํㅡㄹ ENEMET - Encontro Nacional de Estudantes de Engenharia Metalúrgica, de Materiais e de Minas, 21 a 25 de julho de 2014, São Paulo, SP, Brasil.
} 
Tabela 5. Arranjo Ortogonal L9 para os Parâmetros e Níveis dos Experimentos

\begin{tabular}{c|c|ccc}
\hline EXPERIMENTOS & $\begin{array}{c}\text { SEQUÊNCIA } \\
\text { EXPERIMENTAL }\end{array}$ & $\begin{array}{c}\text { NÚMERO DE } \\
\text { PASSES }\end{array}$ & $\begin{array}{c}\text { TAXA DE } \\
\text { ALIMENTAÇÃO }\end{array}$ & $\begin{array}{c}\text { FLUXO DE } \\
\text { NITROGÊNIO }\end{array}$ \\
\hline 1 & 6 & 4 & 30 & 10 \\
\hline 2 & 1 & 4 & 40 & 12,5 \\
\hline 3 & 5 & 4 & 50 & 15 \\
\hline 4 & 2 & 8 & 30 & 12,5 \\
\hline 5 & 4 & 8 & 40 & 15 \\
\hline 6 & 3 & 8 & 50 & 10 \\
\hline 7 & 9 & 12 & 30 & 15 \\
\hline 8 & 7 & 12 & 40 & 10 \\
\hline 9 & 8 & 12 & 50 & 12,5 \\
\hline
\end{tabular}

\subsection{Análise dos Revestimentos}

As imagens para análise e medidas das espessuras foram capturadas através de um microscópio ótico, marca Olympus BX5, localizado no Laboratório de Materiais e Superfícies (LAMATS) da UFPR. Esse equipamento consegue capturar imagens com ampliação de 200x a 1000x e fazer a medição de espessura do revestimento.

\section{RESULTADOS E DISCUSSÃO}

\subsection{Deposição do Revestimento por Aspersão Térmica}

A Figura 1 apresenta o aspecto dos revestimentos aspergidos. É possível observar uma área do substrato esbranquiçado, essa é a área coberta com o alumínio aspergido no processo, sendo evidente que para cada experimento foi obtido uma característica diferente de revestimento, o que é inerente das diferentes combinações de parâmetros utilizados.

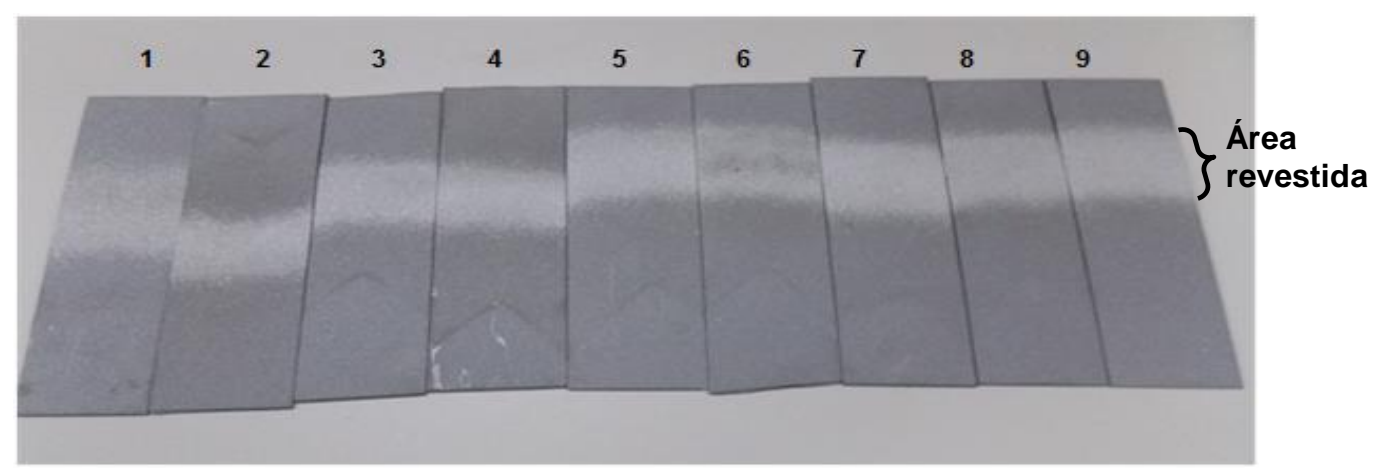

Figura 1 - Substratos Aspergidos dos 9 Experimentos

Em seguida cada corpo de prova foi cortado em 2 partes centrais iguais totalizando 6 metades para cada experimento. A Figura 2 mostra os corpos de prova após embutimento.

\footnotetext{
* Contribuição técnica ao 69ำ Congresso Anual da ABM - Internacional e ao 14ํㅡㄹ ENEMET - Encontro Nacional de Estudantes de Engenharia Metalúrgica, de Materiais e de Minas, 21 a 25 de julho de 2014, São Paulo, SP, Brasil.
} 

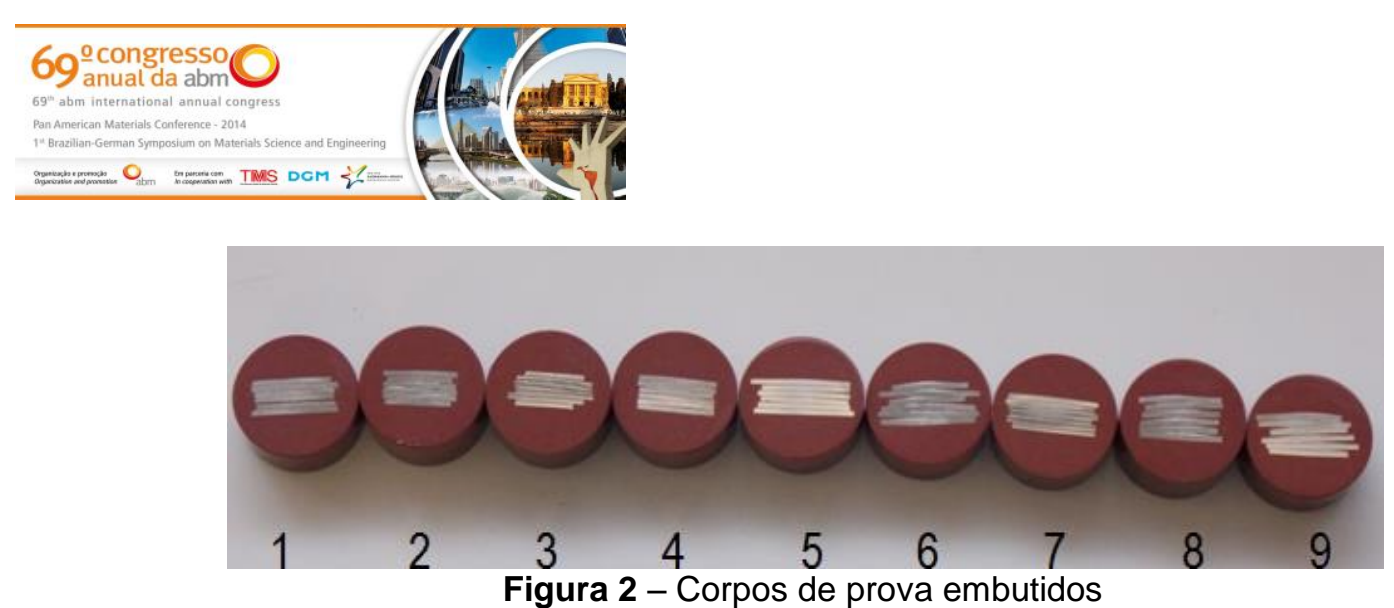

Na Figura 3, pode-se observar a separação das 3 réplicas de cada experimento. Para cada uma das metades embutidas, foram retiradas 3 medidas de espessura.

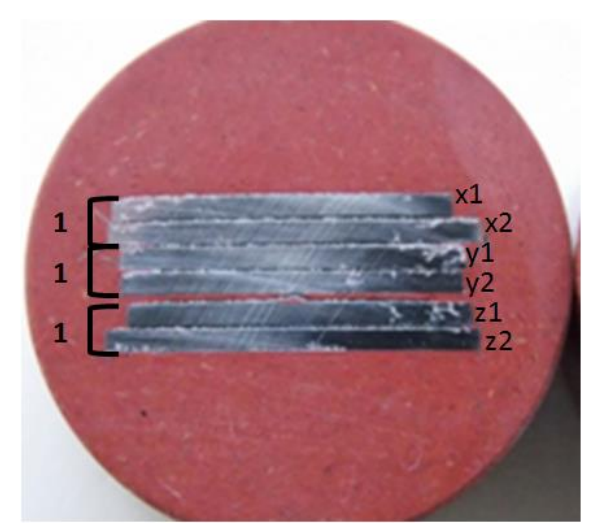

Figura 3 - Réplicas de cada experimento

\subsection{Análise Estatística das Medidas de Espessura dos Experimentos}

A partir dos resultados de medição de espessura, é gerada a estatística descritiva dos dados de espessura apresentada na Tabela 6. Os dados apresentados mostram grande diferença de médias, mediana, mínimo e máximo nos experimentos 6,8 e 9 em relação aos outros experimentos. Essas medidas de posição dos experimentos 6, 8 e 9 demonstram que essa amostra contêm melhores parâmetros que os outros, para se obter a maior espessura de aspersão.

Em relação aos dados de variação dos experimentos somente os experimentos $1 \mathrm{e}$ 6, apresentaram maiores diferenças de desvio padrão e variância que os demais, podem estar ligados aos parâmetros selecionados no processo.

Tabela 6. Estatística Descritiva das Espessuras das Amostras dos Experimentos

\begin{tabular}{l|ccccccccc}
\hline \multirow{2}{*}{ Estatísticas } & \multicolumn{8}{|c}{ Espessura dos Experimentos de 1 a 9} \\
& 1 & 2 & 3 & 4 & 5 & 6 & 7 & 8 & 9 \\
\hline Média & 130,77 & 147,32 & 141,76 & 137,03 & 145,17 & 544,84 & 180,11 & 582,15 & 453,01 \\
\hline StDev & 27,11 & 43,69 & 50,27 & 44,55 & 34,65 & 98,88 & 56,52 & 52,80 & 56,77 \\
\hline Variância & 734,80 & 1909,10 & 2526,70 & 1984,90 & 1200,50 & 9777,40 & 3194,80 & 2787,70 & 3222,80 \\
\hline Mediana & 128,97 & 142,88 & 132,00 & 142,56 & 140,00 & 523,84 & 171,04 & 588,16 & 453,12 \\
\hline Mínimo & 96,96 & 79,04 & 67,84 & 51,20 & 72,00 & 393,60 & 96,00 & 501,76 & 349,44 \\
\hline Máximo & 200,00 & 258,88 & 242,88 & 199,04 & 220,80 & 732,16 & 285,44 & 657,92 & 560,00 \\
\hline
\end{tabular}

Para se interpretar melhor os dados de distribuição das amostras, na Figura 4 é apresentado o gráfico de boxplot. A partir do gráfico verifica-se que o experimento 2

\footnotetext{
* Contribuição técnica ao 69ำ Congresso Anual da ABM - Internacional e ao 14ํㅡㄹ ENEMET - Encontro Nacional de Estudantes de Engenharia Metalúrgica, de Materiais e de Minas, 21 a 25 de julho de 2014, São Paulo, SP, Brasil.
} 


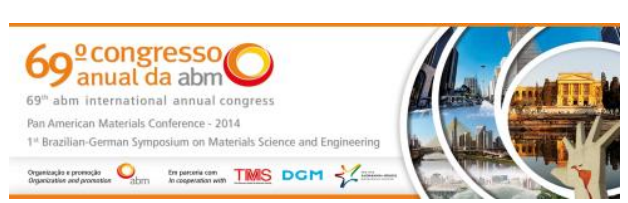

apresenta a existência de um outlier com assimetria positiva, esse dado pode influenciar nas análises posteriores deslocando a média da amostra, assim, esse dado será retirado das analises futuras. O bloxplot mostra também que os experimentos 1, 2, 3, 4, 5 e 7 apresentam dados similares entre as amostras com mediana de espessura inferior aos experimentos 6,8 e 9, demonstrando graficamente que esses 3 grupos de experimentos contêm parâmetros de processo que se destacam aos demais.

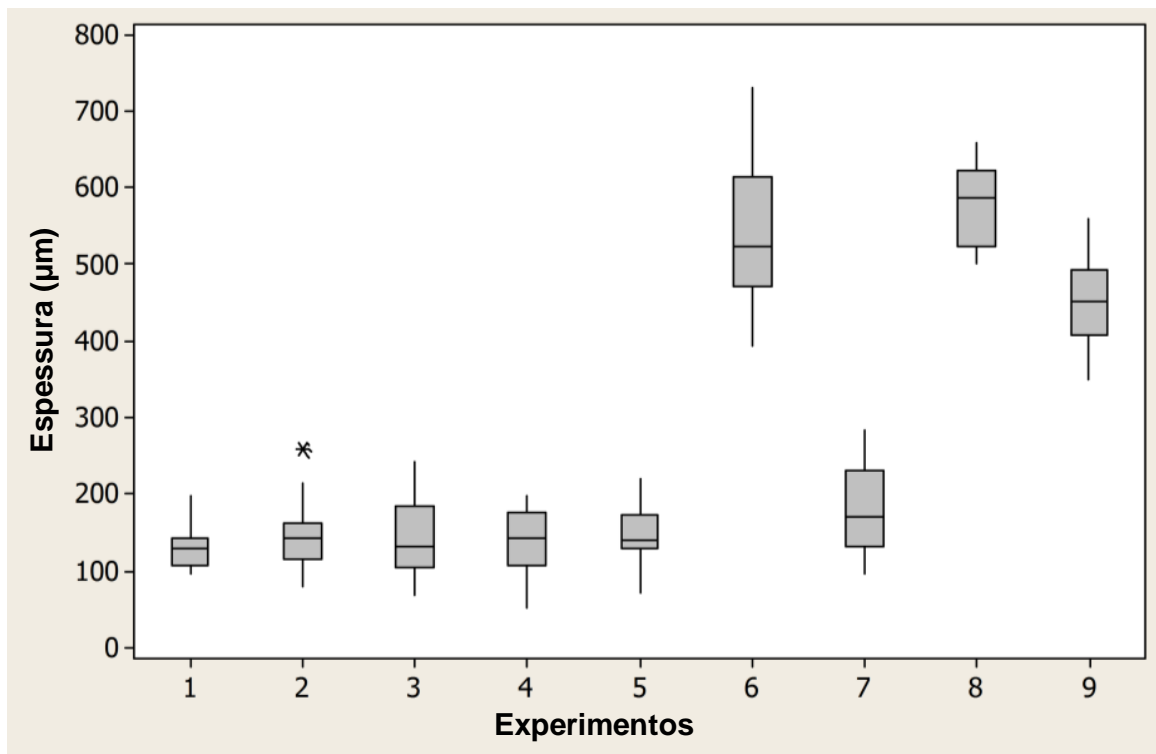

Figura 4. Gráfico Boxplot das Espessuras dos Experimentos

\subsection{Análise de Taguchi}

Com o objetivo de verificar os parâmetros mais influentes no aumento da espessura aspergida, se faz necessário o calculo da relação Sinal/Ruído para o fator "Maior é Melhor". Essa relação verificará quais os níveis dos fatores que contribuem para o aumento da espessura do revestimento no processo de aspersão térmica.

Depois de calculado os efeitos médios dos fatores para a relação $S / R$, é calculado a variação dos fatores pelo intervalo $\Delta$ (Delta) e então ranqueados do maior para 0 menor valor, como apresentado na tabela 7.

Tabela 7. Ranqueamentos da Relação Sinal/Ruído - Maior é Melhor.

\begin{tabular}{lccc}
\hline Nível & $\begin{array}{c}\text { Numero de } \\
\text { Passes }\end{array}$ & $\begin{array}{c}\text { Taxa de } \\
\text { Alimentação }\end{array}$ & $\begin{array}{c}\text { Fluxo de } \\
\text { Nitrogênio }\end{array}$ \\
\hline 1 & 42,17 & 42,12 & 50,46 \\
\hline 2 & 45,74 & 46,87 & 45,53 \\
\hline 3 & 50,68 & 49,60 & 42,60 \\
\hline$\Delta$ (Delta) & 8,51 & 7,48 & 7,87 \\
\hline Ranq. & 1 & 3 & 2 \\
\hline
\end{tabular}

A tabela 7 de ranqueamento apresenta o nível de variação de cada fator. Com isso, é possível verificar a partir da relação $S / R$ que o fator que mais influência nos valores de espessura é o Fator A - Número de Passes, seguido pelo Fator C - Fluxo de Nitrogênio e por ultimo o Fator B - Taxa de Alimentação de pó.

\footnotetext{
* Contribuição técnica ao $69^{\circ}$ Congresso Anual da ABM - Internacional e ao 14ํㅡㄹ ENEMET - Encontro Nacional de Estudantes de Engenharia Metalúrgica, de Materiais e de Minas, 21 a 25 de julho de 2014, São Paulo, SP, Brasil.
} 


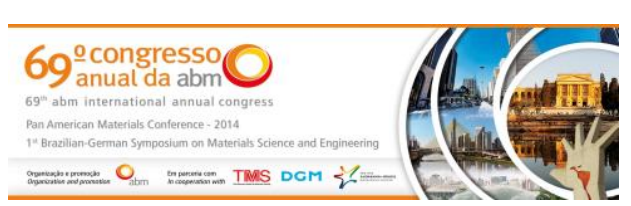

Na Figura 5, observa-se pelo gráfico dos efeitos médio sobre a relação $S / R$, que a melhor configuração proposta pelo método para se maximizar a espessura de deposição no processo é: Número de Passes $=12$; Taxa de alimentação $=50 \mathrm{e}$ Fluxo de Nitrogênio $=10$

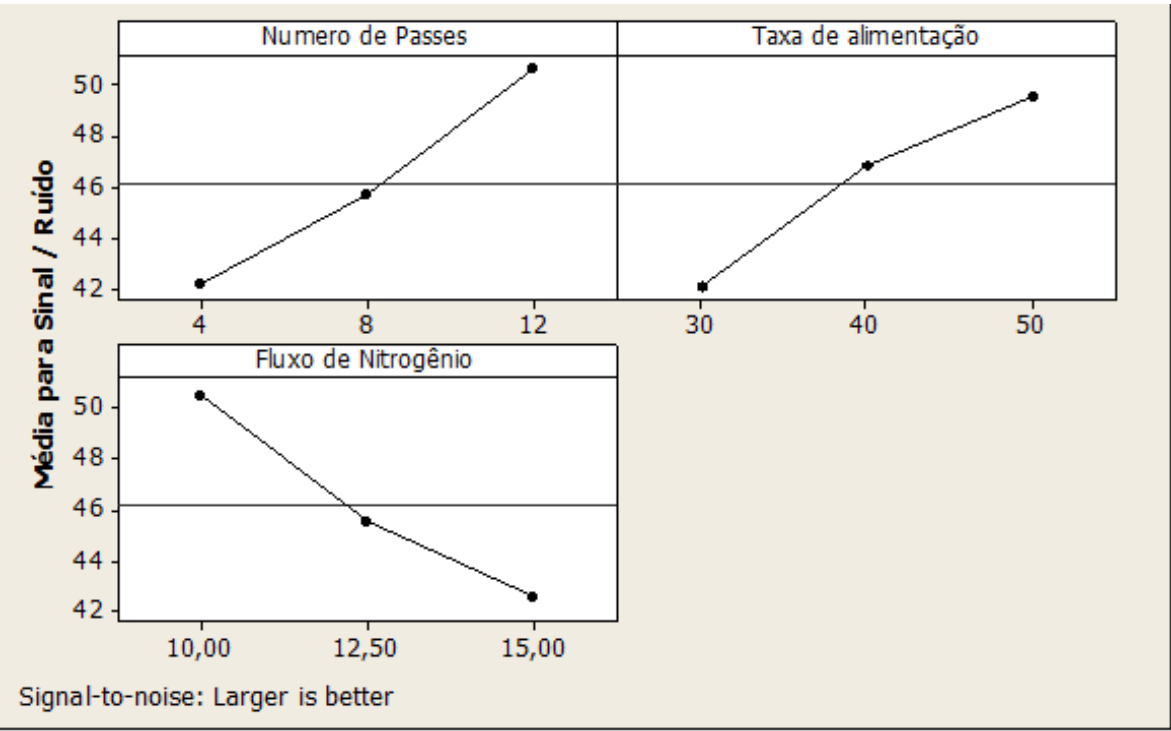

Figura 5. Gráfico dos Efeitos Médio Sobre a Taxa Sinal/Ruído

\section{CONCLUSÃo}

Por meio do delineamento de experimento de Taguchi foi possível investigar a influência dos parâmetros no processo de aspersão térmica por chama a pó no estudo da melhor espessura resultante de deposição nas peças.

A análise dos dados pelo método de Taguchi apresentaram a melhor combinação de parâmetros no que se refere a maior espessura no processo de aspersão térmica por chama a pó. A configuração ideal dos fatores gerada foi: Número de Passes: 12; Taxa de alimentação: $50 \mathrm{~g} / \mathrm{min}$; e Fluxo de Nitrogênio: $10 \mathrm{SCFH}$.

Com relação ao efeito médio dos fatores de relação sinal ruído, o Fator A "Numero de Passes" apresenta maior variação sobre o resultado da espessura, seguido pelo Fator C "fluxo de Nitrogênio" e por ultimo pelo Fator B "Taxa de Alimentação".

Sendo assim, os parâmetros em ordem de maior influência no aumento da camada de deposição, ou seja, espessura de revestimento são: o número de passes, seguido dos padrões de máquina, fluxo de nitrogênio (gás de arraste) e da taxa de alimentação.

\section{Agradecimentos}

Os autores agradecem ao Laboratório de Aspersão Térmica e Soldagens Especiais (LABATS) da UFPR por possibilitar a deposição por aspersão térmica. A UTFPR Campus Cornélio Procópio e a Fundação Araucária, pelo apoio financeiro. Ao programa de pós-graduação PG-MEC (Programa de Pós-Graduação em Engenharia Mecânica).

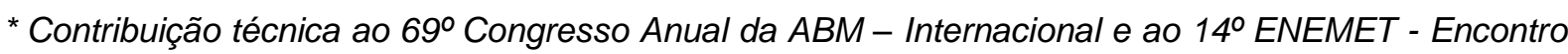
Nacional de Estudantes de Engenharia Metalúrgica, de Materiais e de Minas, 21 a 25 de julho de 2014, São Paulo, SP, Brasil.
} 


\section{REFERÊNCIAS}

1 Silva JGCD. Estatística Experimental: Planejamento de Experimentos. Universidade Federal de Pelotas. Pelotas, 2007: 511. 2007.

2 Marques, P. V. Aspersão Térmica. Infosolda, 2003. Disponivel em: $<$ http://www.infosolda.com.br/images/Downloads/Artigos/processos_solda/aspersotermica.pdf>. Acesso em: 25 jun. 2013.

3 Sulzer. 5MPE Powder Feeder - Manual Assembly. 1. ed. Wearbury, NY:., v. único, 2004.

4 Syrcos GP. Die Casting Process Optimization Using Taguchi Methods. Journal of Materials Processing Tecnology, 2003.

5 Wu DH, Chang MS. Use of Taguchi Method to Develop a Robust Design for the Magnesium Alloy Die Casting Process. Materials Science e Engeneering A, 2004.

* Contribuição técnica ao 69 Congresso Anual da ABM - Internacional e ao 14ํㅡㄹ ENEMET - Encontro Nacional de Estudantes de Engenharia Metalúrgica, de Materiais e de Minas, 21 a 25 de julho de 2014, São Paulo, SP, Brasil. 